\title{
DELTA SPOTS AND GREAT FLARES
}

\author{
HAROLD ZIRIN and MARGARET A. LIGGETT \\ Big Bear Solar Observatory, California Institute of Technology \\ Pasadena, California 91125
}

\begin{abstract}
Using eighteen years of observations at Big Bear, we summarize the development of $\delta$ spots and the great flares they produce. We find $\delta$ groups to develop in three ways: eruption of a single complex active region formed below the surface, eruption of large satellite spots near (particularly in front of) a large older spot, or collision of spots of opposite polarity from different dipoles. Our sample of twenty-one $\delta$ spots shows that once they lock together, they never separate, although rarely an umbra is ejected. The $\delta$ spots are already disposed to their final form when they emerge. The driving force for the shear is spot motion, either flux emergence or the forward motion of $p$ spots in an inverted magnetic configuration.

We observe the following phenomena preceding great flares:

1. $\delta$ spots, preferentially Types 1 and 2 .

2. Umbrae obscured by $\mathrm{H} \alpha$ emission.

3. Bright $\mathrm{H} \alpha$ emission marking flux emergence and reconnection.

4. Greatly sheared magnetic configurations, marked by penumbral and $\mathrm{H} \alpha$ fibrils parallel to the inversion line.
\end{abstract}

We assert that with adequate spatial resolution one may predict the occurrence of great flares with these indicators.

\section{INTRODUCTION.}

Neophytes to solar observing are often disappointed to follow large sunspots for days without observing any flares. It was Kunzel (1960) who first defined the $\delta$ configuration and showed that it was associated with a high frequency of flares. But Kunzel et al. (1961) soon found $\delta$ groups that were not active. Since then we have found other harbingers of high flare activity: inverted polarity, the reversal of the normal Hale-Nicholson polarity (Smith and Howard 1968; Zirin 1970a) and persistency, the tendency of flares to occur where there already have been many.

Zirin (1970a) reviewed the first few years of medium resolution data taken with a small telescope in Pasadena, emphasizing polarity inversion and flux emergence. Tanaka (1975) showed that $90 \%$ of $\delta$ groups with inverted polarity were associated with great activity, and gave detailed descriptions of the evolution of these groups. Other ideas on flare prediction are found in the same volume (Donnelly 1975), but the resolution of the data used was too low for successful prediction. Sawyer et al. (1986) recently discussed many of these problems of flare prediction. 
Because the $\delta$ groups are so significant, we studied the evolution of $21 \delta$ groups to understand how they were formed. We also studied the behavior and preflare conditions of a number of great flares observed at BBSO; we found that all occurred in $\delta$ groups, but that other characterisitics were present as well. Since $\delta$ groups are intimately tied to great flares, we will discuss them first.

\section{FORMATION OF HIGHLY ACTIVE $\delta$ CONFIGURATIONS.}

Kunzel defined the $\delta$ configuration to be sunspots of opposite magnetic polarity in a single penumbra. This is often used to refer to a penumbra with a small satellite spot of opposite polarity (Rust 1968) which is a common source of flares. But we find that great activity occurs only when substantial spots are involved in both polarities of the $\delta$ configuration, and we focus our attention on these cases.

We find we can classify $\delta$ spots in three distinct classes according to their formation:

1. A complex of spots emerging all at once with different dipoles intertwined. We call these the "island" $\delta$ configurations. They are quite compact, with a large umbra, usually $p$ polarity, surrounded by bright $\mathrm{H} \alpha$ emission.

2. A single $\delta$ group, as Type 1, but formed in two stages, with large satellite dipoles emerging in the penumbra of a large $p$ spot.

3. A $\delta$ configuration formed by collision between two separate but growing bipolar spot groups, the leader of one colliding with the follower of the other.

The prototype of the island $\delta$ group is (Figure 1) the Aug 1972 region (Zirin and Tanaka, 1973), which formed 15 days earlier with inverted polarity, and was always a tight complex of spots. A large and growing $p$ spot was trying to push its way through large $f$ spots. But the new spot and its sibling were already in the region; this was no random collision. MtW 19427 (Figure 2) is an example of Type 1 which was not island-like, but formed from many dipoles (Tanaka 1975) erupting at once. The largest had a $p$ spot moving directly through an $f$ spot, producing an extended series of flares along the sheared boundary. As is often true in these complex groups, we cannot identify the sibling of the moving $p$ spot.

Type 2 is illustrated in Figure 4 and also by MtW 19469 of Sept 1974 (Tanaka 1975). Large new dipoles erupt near a large $p$ spot and the shear occurs as they grow up against the main spot. If that spot is small (as in Zirin 1983) the flares are moderate; if it is large, then big flares may occur. The Aug 1972 group formed with a dominant $p$ spot surrounded by small $f$ spots; when it returned 16 days later there was a dominant $f$ spot with a moving $p$ spot. The umbrae in these groups change rapidly. There is a separate sub-class of the second type of $\delta$ spot where the main $p$ spot is part of an otherwise normal group. In MtW 21030 (Nov 1979), continuous flux eruption with big flares occurred around the lead spot MtW 21030 (Patterson and Zirin 1981; Zirin et al. 1982). These can usually be recognized by a filament 


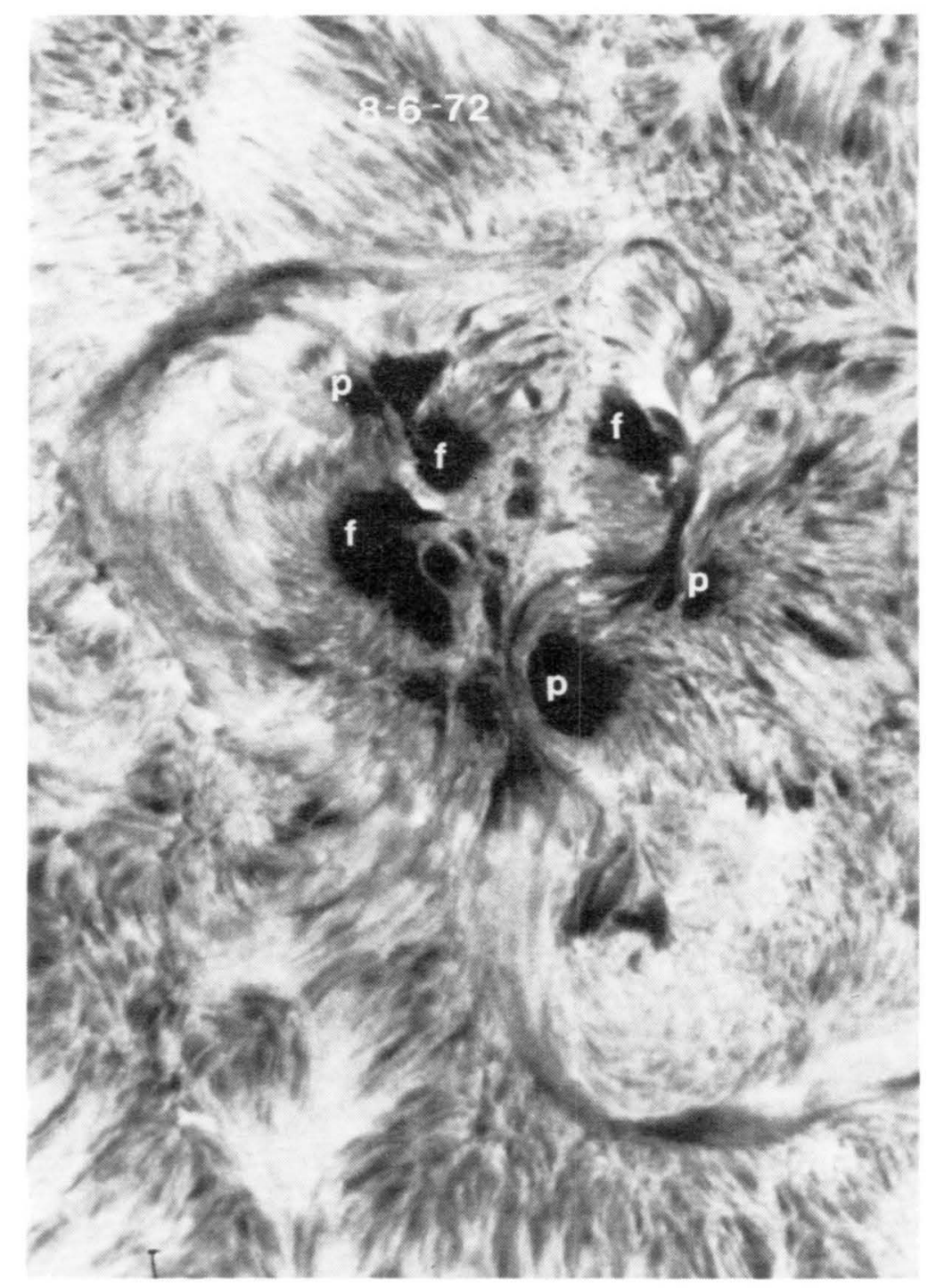

Fig. 1. The MtW 18935, Aug 61972 , one of the most active regions ever (Zirin and Tanaka, 1973). Preceding and following polarity are indicated. This frame is in the blue wing of $\mathrm{H} \alpha$, and in this and other such frames the penumbrae are still somewhat obscured. The region formed on July 12 as a large $p$ spot surrounded by small $f$ spots, and rotated off with a blaze of surges. There was constant flux emergence within a tight circle, and the main (lowest) $p$ spot pushed continually through the $f$ polarity to the $\mathrm{W}$ (left) of the inversion line. This can be seen through the semicircular form of the filament to the $\mathrm{W}$ of it. By Aug 7 the top $f$ spot had disappeared and a new $f$ spot had appeared $\mathrm{W}$ of the filament. $\mathrm{S}$ top, $\mathrm{W}$ left on all figures. 


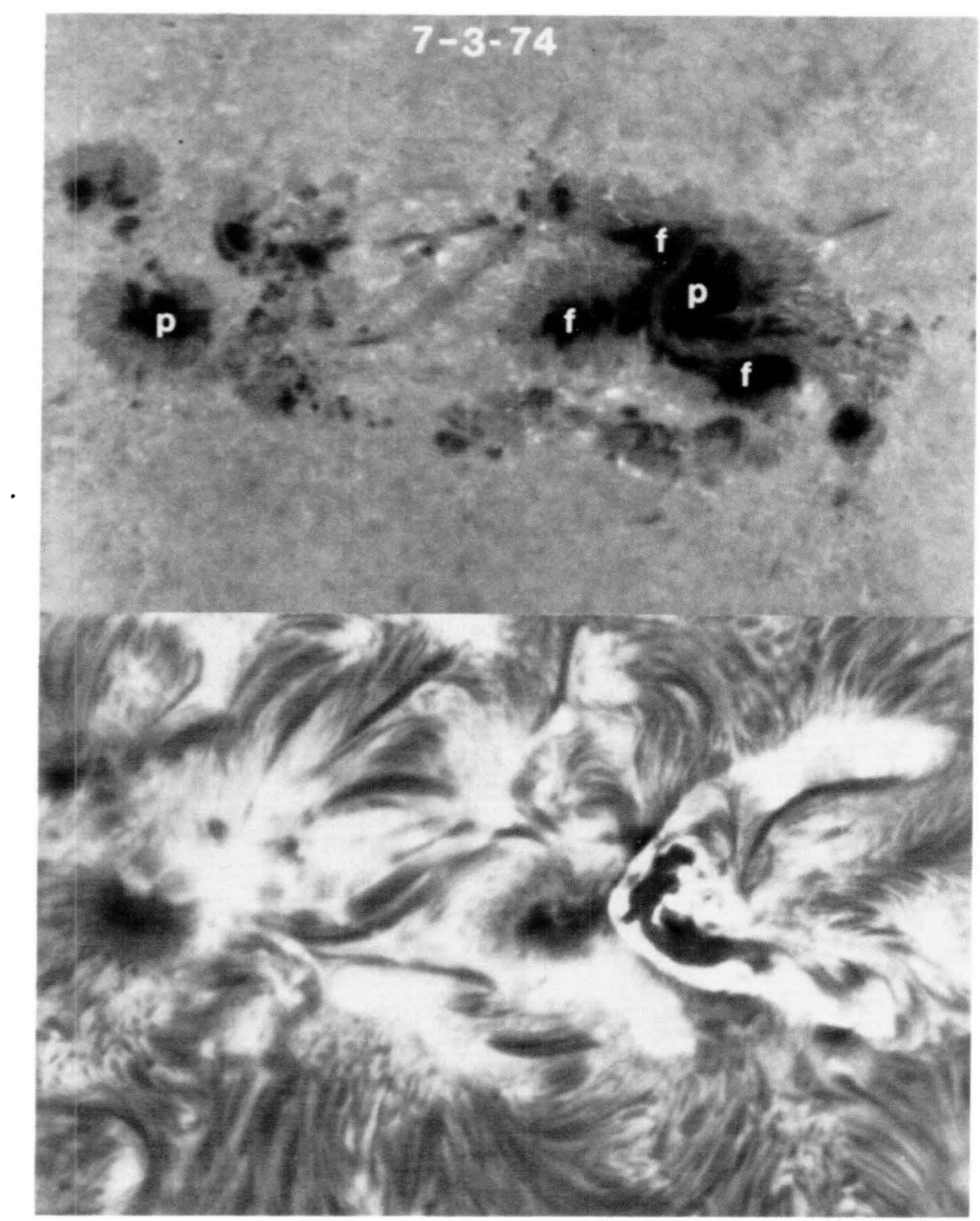

Fig. 2. MtW 19427, July 3, 1974, which had more but somewhat smaller flares than MtW 18935 (Tanaka 1975). This complex of many spots emerged at once. Above: $\mathrm{H} \alpha+1 \AA$; below: $\mathrm{H} \alpha$. The large $p$ spot at right emerged with the cluster of $f$ spots and immediately began to grow and push through them. The flares occurred on the inversion line. Note that almost all the spots are obscure in $\mathrm{H} \alpha$. Because all these spots emerged at once we place this group in the first class of $\delta$ groups. 


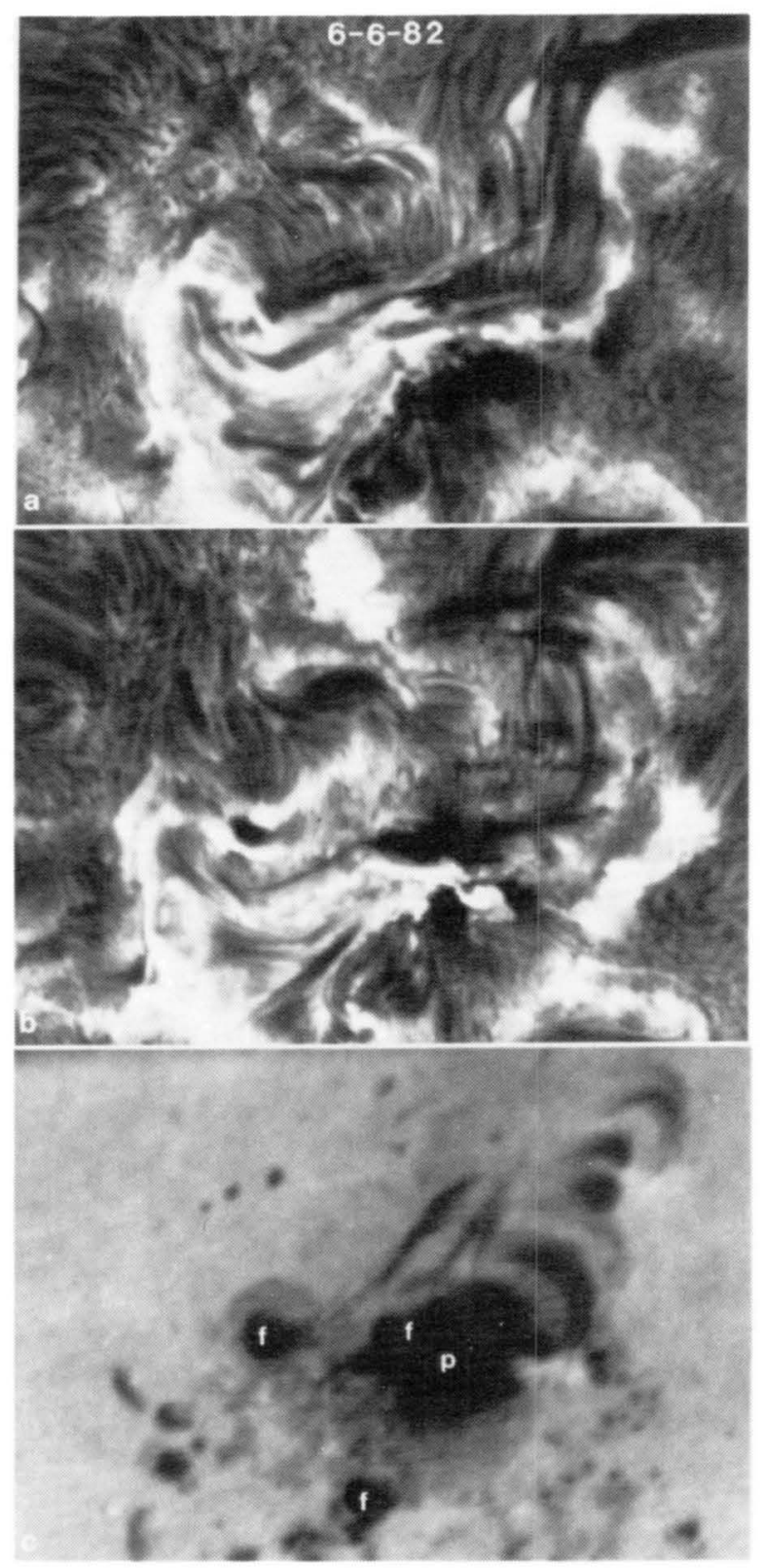

Fig. 3. Before (a) and after (b) the great flare of July 6, 1982 in MtW 23169 (Tanaka and Zirin, 1985). Before the flare, the spots, especially the moving $f$ spot at left, were covered by $H \alpha$ emission. After the flare, most of them was visible. (c) in the red wing, shows the post-flare loops connecting the moving spot with the following part of the region. The main spot that it formerly connected to is now connected to adjacent new spots. 


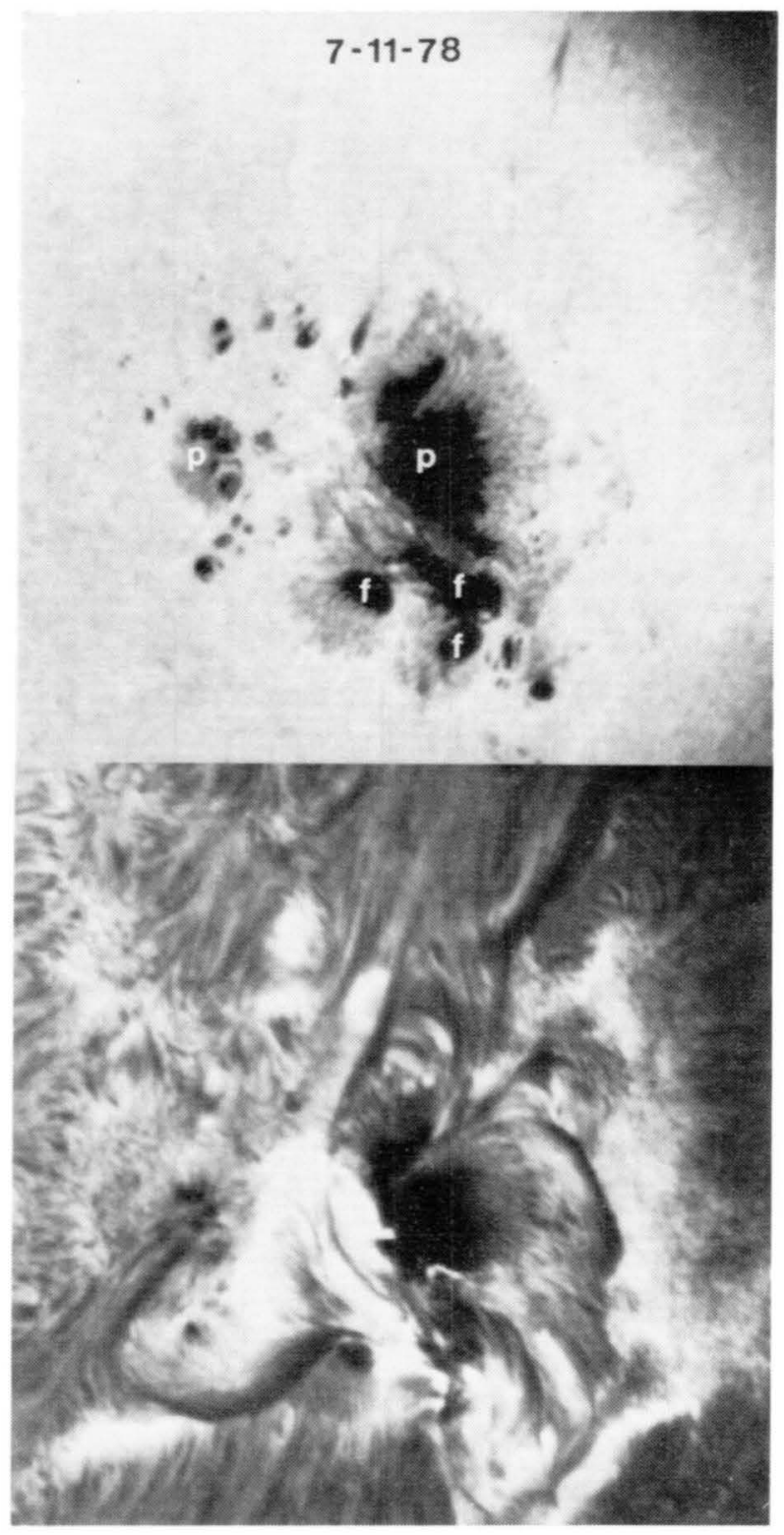

Fig. 4. MtW 15403, July 11, 1978 group. The $f$ spot closest to the main umbra is part of an EFR, probably paired with the $p$ spot at the left, which grew rapidly till it was twice the present size. In the centerline frame below the satellite spots are all obscured. 
winding around bright plage at the leading edge of the main $p$ spot and high $\mathrm{H} \alpha$ brightness in front of it. Continuum frames reveal big satellite spots obscured by $\mathrm{H} \alpha$ fibrils. Because the flare source is in front of the $p$ spot, these regions are prolific sources of Moreton waves.

The details of the formation of $\delta$ spots of Type 3 by spot collision are hard to understand (Zirin 1983). When spots are pushed together, it is reasonable that the shear should occur. But when they slide past one another, first connections must be made which then must be stretched by the continuing motion. The two steps seem contradictory. It may be that subsurface reconnection has occurred, and the spots are pulled against one another by the tension in the subsurface connection. As Kunzel et al. found, these $\delta$ spots are not so active. Examples of Type 3 are given by Kunzel et al. (1961) and Tang (1983).

We found the following characteristics to be common to all the $\delta$ spots that we studied:

1. They form from opposite polarity spots from different dipoles.

2. They rarely last more than one rotation and are shorter-lived than other spots of the same size, but new $\delta$ spots may emerge in the same complex (April-May 1984, MtW 24030, 24057).

3. The polarity is generally inverted as compared to the Hale-Nicholson Law.

4. They are never observed to separate (although spots may be ejected), but die out locked together.

5. Except in the final decay state, the field lines connecting the spots are sheared and run parallel to the inversion line.

Figure 6 shows a rare exception to the general rule that the $\delta$ spot forms only out of a pair of sunspots from opposite dipoles. In this case the spots never separated. If separate dipoles were not required, any dipole pair could form a $\delta$ spot by simply shrinking back together. The energetic behavior of the $\delta$ configuration tells us that a lower state is achieved by the reconnection of the two dipoles, while no energy is liberated by a shrinking of a simple dipole. Because Types 1 and 2 erupt in the same place, and Type 3 requires large dipoles that are not close by mere accident, the $\delta$ configuration must be the product of a subsurface phenomenon.

The energy of the $\delta$ spots must come from their shortening magnetic connection and the shear imposed on that configuration by spot motions. As the spots lock closer and closer together, energy must be released. Two big spots separated by only a few thousand kilometers cannot have a direct potential field connection between them, but the field lines must go up and down in some sharp and twisted form. The solution which the sun follows is for these field lines to lie parallel to the boundary line. Above that level, there are normal potential-like loops. This happens either if the spots are pushed together or if they slide past one another. It is unlikely that 


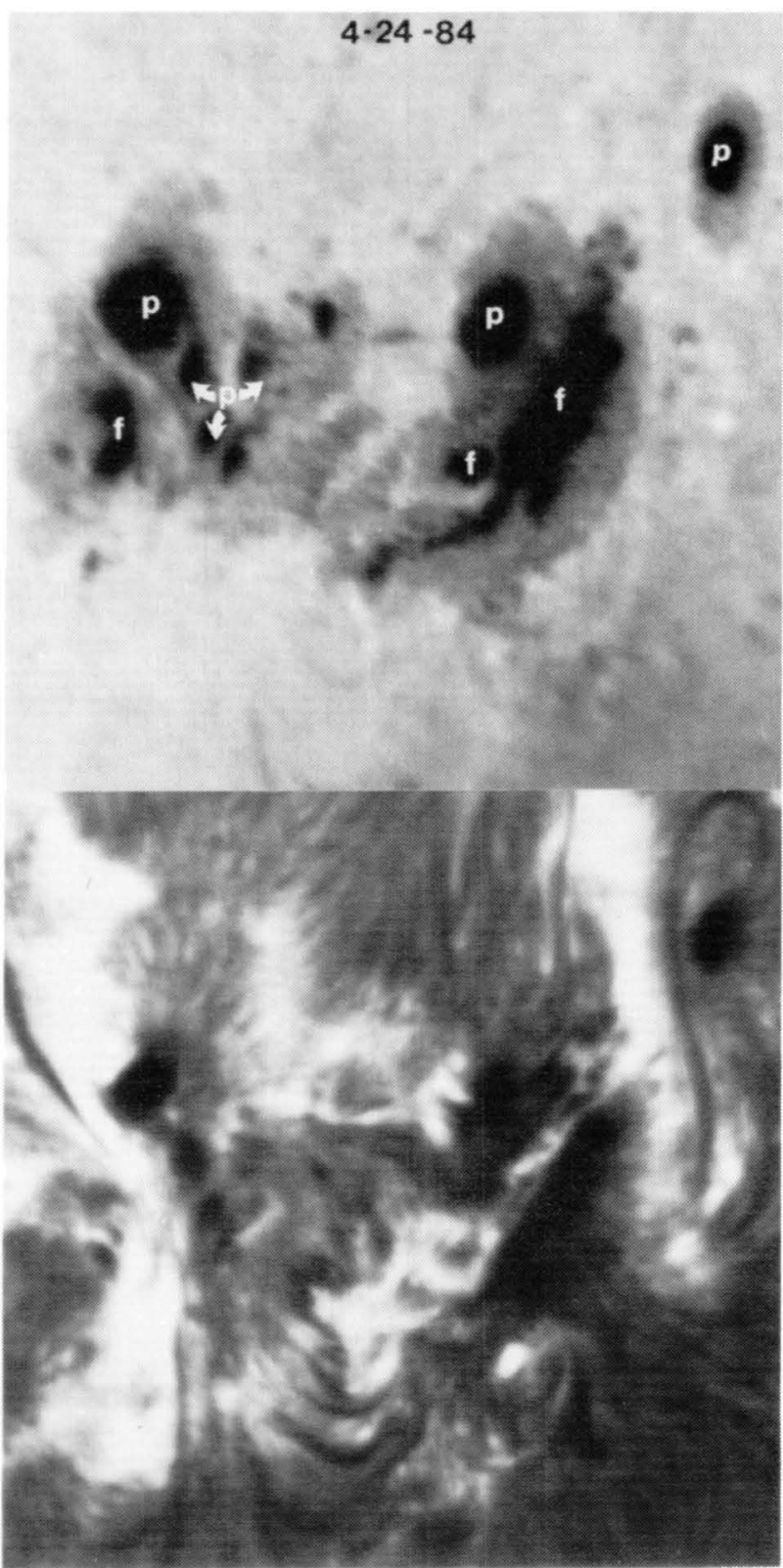

Fig. 5. The April 24, 1984 group some hours before the great flare, which occurred in the left $\delta$ spot cluster. 


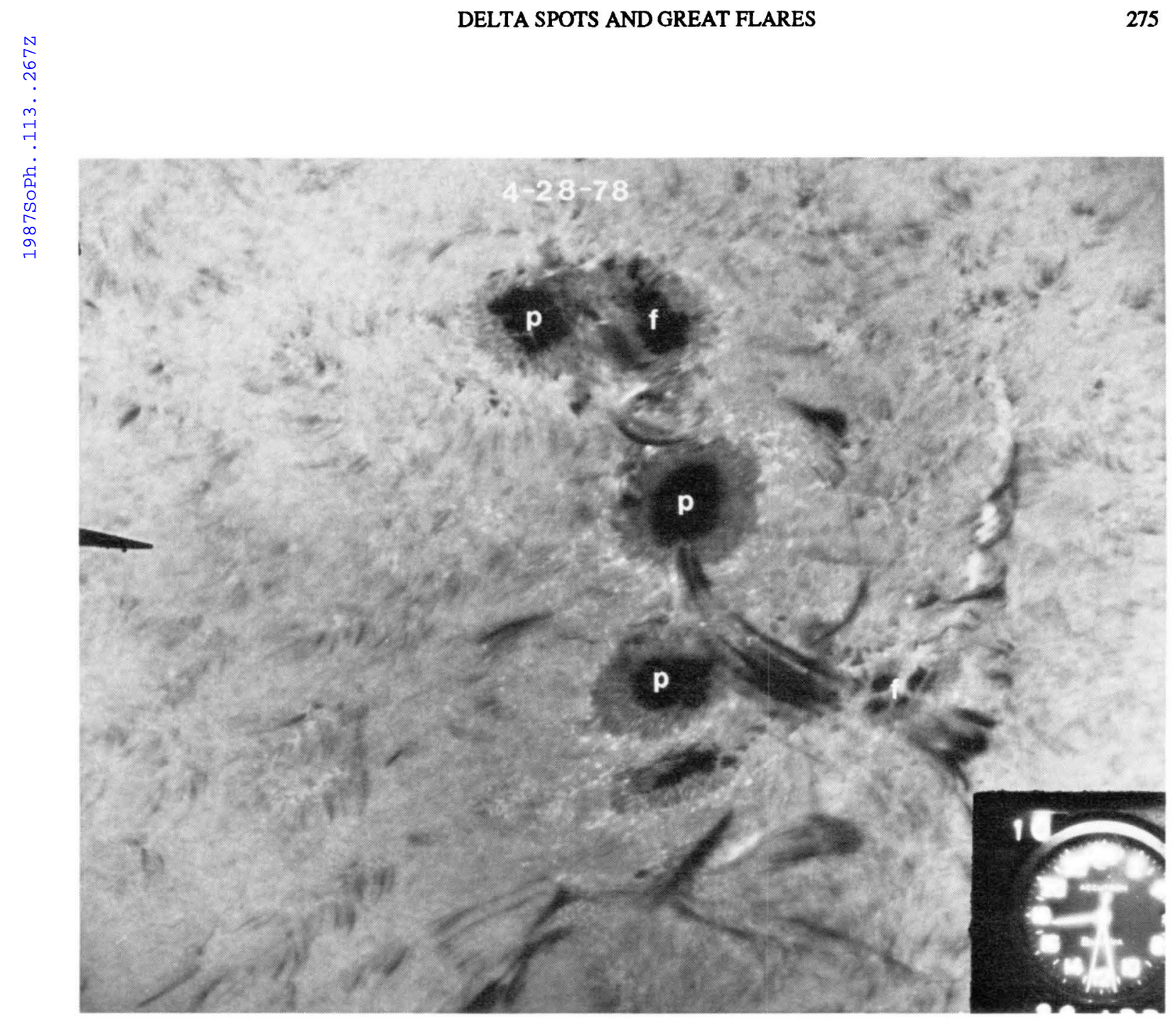

Fig. 6. The exception to delta formation by separate dipoles: The $p$ and $f$ spots at the top emerged on the preceding day and never separated. They showed little activity. 
such spots can ever connect directly, and we find that the shear boundary persists. During most of the Aug 1972 flares the spots were still separated by shear and the postflare loops were quite high, until the Aug 7 event, which was followed by relatively short flux loops connecting the $\delta$ spots.

As the $\delta$ spots decay, direct magnetic connections occur, and the spots are connected by short, direct flux loops. We have never succeeded in observing the disappearance of such a region, but we are convinced that they did not ever separate, but subsided below the photosphere.

In two of the twenty-odd $\delta$ spot groups we studied, a sunspot was ejected. In Figure 7 we see MtW 21567 (Zirin and Tanaka, 1981) and in Figure 3, MtW 23169 (Tanaka and Zirin 1985), a single spot left the $\delta$ configuration, but a $\delta$ configuration remained behind. In Figure 7 the $\delta$ spot moved westward and the spot FA was left behind; we observed one sizable flare. In MtW 23169, the moving spot produced a series of flares. Some occurred because the spot was moving through other magnetic fields, but some, especially the June 6 event, involved reconnection of the largest spot to new spots near it, while the moving spot reconnected to other $f$ poles as evident in the last frame of Figure 3.

Why do $\delta$ groups behave as they do? Even though the emergence of a sunspot takes a few days, these flux loops have probably existed below the surface for some time, and were not just formed in the last few days before eruption. This should have been sufficient to eliminate their complexities. Some possibilities are:

1. Sunspots of regular form are produced by the general process of the sunspot cycle, but a small fraction are twisted up into $\delta$ spots in the convective zone.

2. Conversely, the active regions are all complex when created, but most of that complexity is lost to subsurface flares and reconnection as they rise to the surface. Then the force that pulls the $p$ spots forward orders them according to the Hale-Nicholson law. The complex $\delta$ configurations we see are the big groups that got through the ordering process.

3. The solar surface presents a particular set of circumstances (such as low conductivity compared to the subsurface layers), which allows magnetic merging to take place more readily than in the interior.

We cannot decide between these possibilities, but must search the observations for a choice.

\section{CHARACTERISTICS OF THE OCCURRENCE OF GREAT FLARES.}

It is well established that flares are associated with large sunspot groups and likely to occur in regions that have already had many flares. But the latter, while useful in prediction, tells us little about why the flares are occurring, and the former is not always true. All of the great flares we have observed at Big Bear have occurred in 


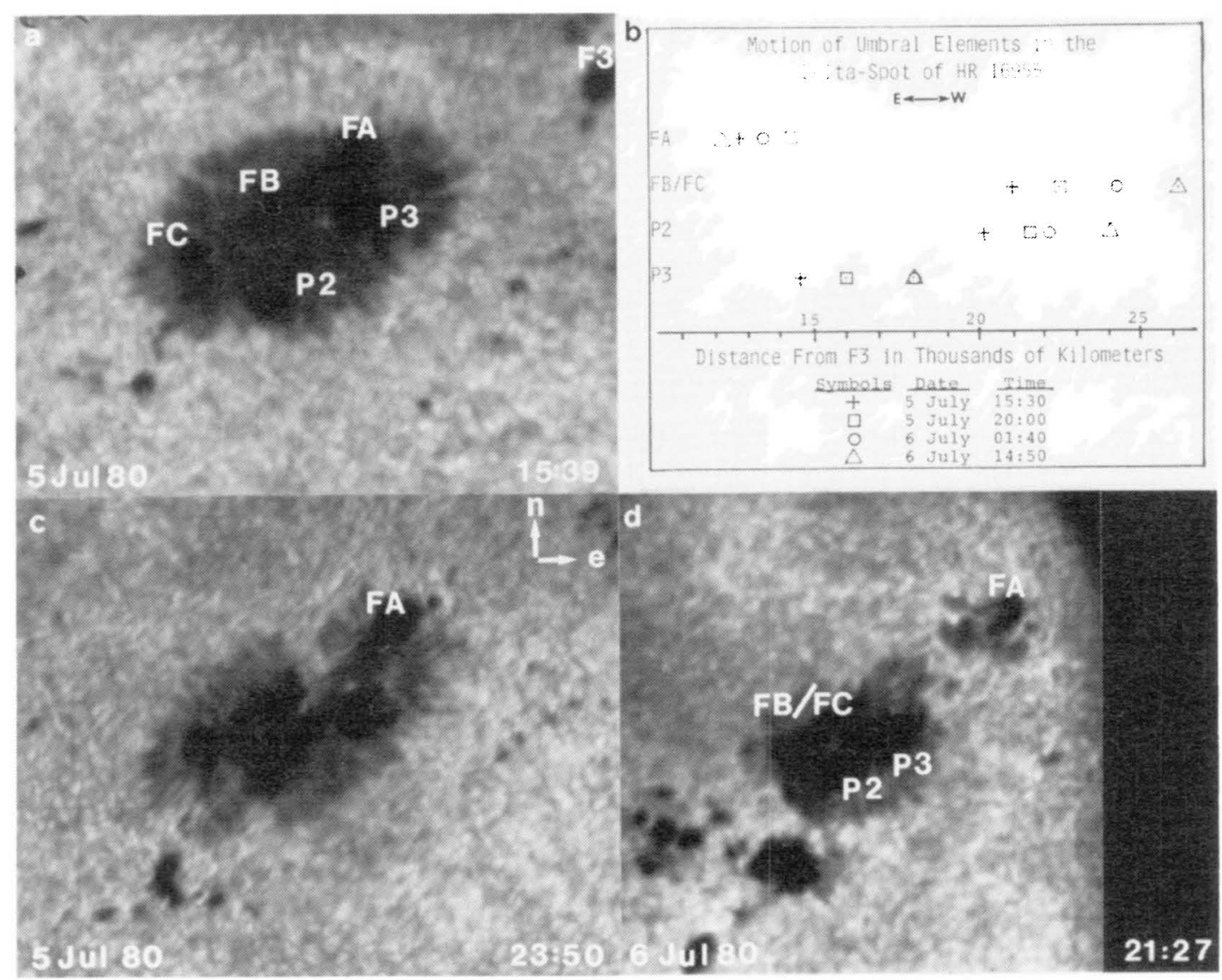

Fig. 7. Ejection of the spot FA from MtW 21567. The relative positions are plotted in (b) but inverted from the pictures. The spot FA did not move relative to F3, while all the other spots of the $\delta$ configuration moved westward. Other spots were emerging to the $\mathrm{W}$. There was a sizable flare at 22:35 UT. 
$\delta$ spots, the largest flares occurring in Types 1 and 2 . We have found the following characteristics to precede all of the great flares (in addition to the $\delta$ configuration):

1. Umbrae obscured by $\mathrm{H} \alpha$ emission.

2. Bright $\mathrm{H} \alpha$ emission marking flux emergence and reconnection.

3. Greatly sheared magnetic configurations, marked by penumbral and $\mathrm{H} \alpha$ fibrils parallel to the inversion line.

These characteristics are all symptoms; the cause is invariably sunspot eruption, growth or motion blocked by other spots.

Obscuration of the umbra by $\mathrm{H} \alpha$ emission or absorption is invariably associated with flare activity. Figure 3 is an excellent example of this though it may be seen in all the $\mathrm{H} \alpha$ centerline figures presented here. On many occasions (such as the great flare of September 10,1974 ) where the main $p$ spot is not covered by emission, the flare occurs in a large complex of $\delta$ spots nearby, which are completely invisible in $\mathrm{H} \alpha$ line center but are readily seen in continuum pictures.

The significance of the overlying $\mathrm{H} \alpha$ material is that the vertical magnetic field of a simple umbra cannot support higher density material. The overlying material can only be there if supported by a horizontal magnetic field directly above the spot. This in turn implies that the field has a curl and is not current-free. In these cases the $\mathrm{H} \alpha$ fibrils and white-light penumbral structure always lie parallel to the inversion line, at right angles to their normal radial direction. If two large spots are connected along such a sheared inversion line, much of the spot flux is crowded into this corridor, and the transverse fields may be quite intense. It seems more likely that this configuration forms when an umbra has emerged in or moves into a region with strong overlying field, creating a current sheet between the new field and old that lies directly above the new umbra.

Bright $\mathrm{H} \alpha$ emission in an established active region is the simplest mark of activity. It should be distinguished from the bright $\mathrm{H} \alpha$ of an EFR. When we see this brightening it means an EFR is coming up in an active region, and in most cases this will lead to shear, reconnection and flares. $\mathrm{H} \alpha$ brightening is often dismissed as an unsophisticated indicator, perhaps because it requires little equipment; but it is in fact the most useful for the following reason: While a magnetogram gives us an image at a single point in time, the $\mathrm{H} \alpha$ emission depends on the local temporal evolution, a critical factor. A glance at the top frame of Figure 2 shows us that there is great shear; a glance at the lower frame ( $\mathrm{H} \alpha$ centerline) tells us that the shear is increasing. When the spots stop emerging or moving into one another or reconnecting, the $\mathrm{H} \alpha$ brightness falls.

The preflare situation on April 24, 1984 (Figure 5) is a nice example of the significance of $\mathrm{H} \alpha$ brightening. There were two $\delta$ spots, MtW 24029-30, one very large and one more of more ordinary size. From a white-light or off-band frame 
or magnetogram one would normally expect big flares from the great spot at the right. But the $\mathrm{H} \alpha$ frame below shows that spot to be quiet, while bright $\mathrm{H} \alpha$ betrays intense activity in the smaller group at left, with a filament along the inversion line, and that is where the great flare occurred.

The greatest shear seems to occur in the island $\delta$ regions where the spots may be constrained to collide. The flare shear is due ultimately to flux emergence and the consequent spot motion. Since usually only the $p$ spots move, collisions occur when the normal Hale-Nicholson polarity is reversed, which is why "reversed polarity" has been associated with activity. The only significance of reversed polarity is that the normal forward motion of the $p$ spot will lead to collision with an $f$ spot in front of it. If flux emerges in quiet regions, the spots will peacefully spread apart and nothing will happen.

Additional factors associated with flare prediction are as follows:

a. Rapid fluctuation of bright $\mathrm{H} \alpha$ emission and big emerging loops in absorption (Zirin 1970b).

b. Steadily increasing sunspot motion (Tanaka and Zirin, 1985)

c. Enhanced radio brightness.

d. Flux eruptions on leading side of dominant $\mathrm{p}$ spot.

e. A filament crossing a $\delta$ spot.

\section{POSSIBILITIES OF FLARE PREDICTION.}

If the characteristics listed above indeed are true of the circumstances preceding great flares, then we are in a position to predict them if images of adequate resolution are available. The value of the criteria given, which we have used for years, is confirmed by the fact that while observing only a small fraction of the sun we have been able to observe most of the large flares that occurred in our observing window. In reading recent symposium papers (Donnelly 1980) on the subject one is struck by the improbability of comprehension of the situation, let alone forecasting, with the low-quality data generally available with patrol instruments. There is no substitute for sufficient resolution to see the active region evolution and understand what is happening. But we must emphasize that these criteria are only useful in predicting where big flares are likely to occur; if the sun is covered with modest regions, prediction is much more difficult.

While prediction of where a great flare will occur is easy, prediction of when it will happen is another matter. We can reasonably predict the time within days, but anything more accurate is presently impossible. The only known precursor is the well-known lifting of the neutral line filament, which may occur from a few to a hundred minutes before the flare. The role of this lifting in the flare is obviously an indication of the destabilization of the magnetic configuration, and the disappearance of obscuring material suggests that a previously sheared magnetic field 
has now opened up into a potential or at least less sheared one.

\section{SUMMARY.}

All the great flares we observed occurred in $\delta$ configurations. These form in three general patterns: emergence of a single complex spot cluster, or island delta; emergence of large satellite spots very close to a large existing spot; and collision of two distinct bipolar groups. Only the first two produce large flares. $\delta$ spots never separate. Since they are formed by the conjunction of umbrae from different dipoles, they must represent a reconnection to form a lower energy state.

We find the following conditions for the occurrence of large flares:

$-\delta$ configurations of the first two kinds.

- Large spots.

- Many previous flares.

- Magnetic shear.

- Steep magnetic gradients.

- Inverse polarity.

- Spots obscured in $\mathrm{H} \alpha$.

- Bright $\mathrm{H} \alpha$ emission.

All of these features are consequences of the first, the locking together of opposite polarity umbrae that emerge in close proximity. The basic process in the $\delta$ spot phenomenon is probably occurring below the surface of the sun. It is necessary to devise observational tests of different models of the development of such activity to understand how it may be predicted. On the other hand, we feel it is possible to use the existence of one or all of the above to make satisfactory flare predictions.

This work was supported by NASA under NGL 05-002-034, the NSF under ATM-8513577, and the AFOSR through AFOSR-87-0034. We are grateful to the Big Bear staff for gathering the data, and to Prof. K. Tanaka for many useful discussions.

\section{REFERENCES}

Donnelly, R. F., ed.: 1975, Solar-Terrestrial Predictions Proceedings Vol. 3, C1. NOAA-ERL.

Kunzel, H.: 1960, Astron. Nachr. 285, 271.

Kunzel, H., Mattig, W., and Schroter, E. H.: 1961, Die Sterne 9/10, 198.

Patterson, A. and Zirin, H.: 1981, Astrophys. J. 243, L99.

Rust, D. M.: 1968, IAU Symp. 35, 7. 
Sawyer, C. S., Warwick, J. W., and Dennett, J. T.: 1986, Solar Flare Prediction, Colorado.

Smith, S. F. and Howard, R. F.: 1968, IAU Symp. 35, 33.

Tanaka, K.: 1975, BBSO Preprint No. 0152.

Tanaka, K.: 1980, in R. F. Donelly (ed.), Solar-Terrestrial Predictions Proceedings Vol. 3, C1. NOAA-ERL.

Tanaka, K. and Zirin, H.: 1985, Astrophys. J. 299, 1036.

Tang, F.: 1983, Solar Phys. 89, 43.

Zirin, H.: 1970a, Solar Phys. 14, 328.

Zirin, H.: 1970b, Solar Phys. 14, 342.

Zirin, H.: 1983, Astrophys. J. 274, 900.

Zirin, H. and Tanaka, K.: 1973, Solar Phys. 32, 173.

Zirin, H. and Tanaka, K.: 1981, Astrophys. J. 250, 791.

Zirin, H. et al.: 1982, Astrophys. J. 259, 392. 


\section{DISCUSSION}

KAI: You talk about the necessary conditions for big flares, for example a cloudy day weekend. In our case, the big flares occur when the equipment didn't work!

ZIRIN: I'm sorry, I forgot about that. As a matter of fact that was fulfilled in the April 24, 1984 flare. One of our cameras was out of film.

STURROCK: Hal, you referred to effect of the subphotospheric field. I'd like to offer a possible interpretation. When you have a single bipolar region appear, just two spots with opposite polarities, then it collapses into a sunspot. You can attribute that to a single flux rope. The lowest-energy state is for the strong field to get up into the corona so the spots are therefore going to move apart, which they do. However, when you see satellite sunspots, what's probably happened, is that one of these field lines has tried to get out and is caught. So you get a little loop hooked down and the surge activity of course keeps on going as this thing tries to get away, until when that loop gets unhooked there's no more satellite sunspot and therefore no more surge activity. My guess is that the delta spot is really the same thing on a bigger scale. You have a lot more flux that's become hooked, so you have below the two umbras of the one spot, you just have a short flux tube holding them together, and that's why they hold together.

ZIRIN: I've thought about that kind of idea of the twisted flux loop. The problem is that would be or in the cases where they come together, but then you have these other ones where one of these things goes right by the other, and if your scenario is right then they would always end up together.

STURROCK: Those that don't stay together aren't connected in this way.

ZIRIN: That may very well be.

SPICER: My feeling is, looking at those pictures, it seems to me that those motions of those spots are determined by something going beneath the photosphere. They're not acting as a surface phenomenon.

ZIRIN: If you look at the motions of the spots, it's about 50/50. Much of their behavior is very systematic as though they are driven by something pulling them from below, particularly the forward motion of the preceding spots. And yet some of these other spots behave like chips on the ocean. They'll separate and, well if they came up as one flux. it's hard to think how they really separate from one another. One end dies off, the other one hooks up with another spot, and they just forget what they ever came up with.

SPICER: Well, reconnection is very probable down subphotospherically probably more likely than above the photosphere. 
ZIRIN: I think also, by the way, if we start looking at the very weak fields - the network fields, the inter-network - those things are on their own. They just come up in one place and they run into another field (this is what Sara has been studying) and they cancel here and they cancel there, and it looks like they know nothing. It's like a field of kelp, but the kelp is no longer attached to anything. I wish we had some more conclusive tests of this idea. Maybe we should focus a little bit more. What argues that they're still attached down below and what argues for flux.

SPICER: Remember the workshop in Big Bear?

ZIRIN: We always see disappearing flux in about 108 of the cases.

SPICER: But that still suggests that something is controlling it.

ZIRIN: And then 908 of the spot groups that come up just go off into the solar wind eventually. They may reconnect with something, but they act as though they forgot where they came from. Maybe that's an exaggeration.

MOORE: I've read papers, and I think even co-authored at least one, where you're quoted as saying that every flare has a filament eruption. What do you think filament eruptions have to do, if anything, with big flares?

ZIRIN: Well, so fax as I can tell, almost all the big flares do have a filament eruption of one sort or another. You can change the nomenclature a little bit to make sure it satisfies the rule, but by and large there's something like that that lifts up and I think the June ones are an examples. How should I know? I can give you some for instances: Model l; the filament is a cool place of low conductivity, it provides a high resistivity, so I can start having connection take place. You can probably prove that's wrong. Model 2; the filament is holding down the lines of force, the lines of force want to go up by magnetic buoyancy and break up this situation. You don't like that one either! It's quite a few tons when you add it up, 1020 tons probably. Model 3; the filament is accidental. We see that every place on the sun where two oppositely directed magnetic fields approach close together we get a magnetic field that runs parallel to the boundary. We get that because if the magnetic field just went across the boundary it would just sink down below the surface. So everything that sank down below the surface until we're left with a sheared boundary. Now almost all those sheared boundaries have filaments in them. Unfortunately you don't know which is the chicken and which is the egg: you don't know whether that filament stabilizes the boundary or whether it's accidental. And I don't know a good test for that.

SPICER: Is it a chicken or a a turkey?

CHAIRMAN: THANK YOU VERY MUCH. 